\title{
MODELACIÓN PRODUCTIVA, ECONÓMICA Y DE GASES DE EFECTO INVERNADERO DE SISTEMAS TÍPICOS DE CRÍA BOVINA DE LA PAMPA DEPRIMIDA
}

\section{PRODUCTIVE, ECONOMIC AND GREENHOUSE GASES MODELLING OF TYPICAL BEEF COW-CALF SYSTEMS IN THE FLOODING PAMPAS.}

\author{
Claudia Faverin ${ }^{1,2}$ *, Franco Bilotto ${ }^{3}$, Catalina Fernández Rosso ${ }^{3}$ y Claudio Machado ${ }^{3}$ \\ ${ }^{1}$ Instituto Nacional de Tecnología Agropecuaria (INTA) Balcarce, Ruta $226 \mathrm{~km} \mathrm{73,5} \mathrm{(CC} \mathrm{276),} 7620$ \\ Balcarce, Argentina. \\ ${ }^{2}$ Facultad de Ciencias Exactas y Naturales (UNMDP), Funes 3350, 7600, Mar del Plata, Argentina \\ ${ }^{3}$ Centro de Investigación Veterinaria de Tandil (FCV, UNCPBA, CONICET y CICBA), ), Paraje Arroyo \\ Seco s/n, Campus Universitario, Tandil, Argentina \\ *Autor para correspondencia: faverin.claudia@inta.gob.ar
}

\section{RESUMEN}

El objetivo de este estudio de simulación fue evaluar la respuesta productiva, económica y de emisión de gases de efecto invernadero (GEI) en establecimientos típicos de la Pampa Deprimida de Argentina. Se utilizó información regional y un modelo de simulación dinámico, bioeconómico y ambiental (SIMUGAN) para evaluar dos tipos de sistemas contrastantes de los 4 identificados en un trabajo previo. Estos dos sistemas se tomaron como línea de base: Productor tradicional de escala media (Tr) y Empresa local tecnificada de escala grande (Te). Ambos escenarios fueron intensificados con la inclusión de festuca bajo buenas prácticas de manejo (5 o $10 \%$ de la superficie del establecimiento) y el adelanto de la edad al primer servicio de las vaquillonas ( 27 a 15 meses) para los planteos con $10 \%$ de la superficie con festuca. Las intensidades de emisión (IE) de GEI por unidad de producto) variaron según el nivel de intensificación de forma similar para Tr y Te (entre 16,4 y $19,9 \mathrm{~kg} \mathrm{CO}_{2}$ eq $\mathrm{kg} \mathrm{PV}^{-1}$ ). El efecto aditivo de la incorporación de festuca a un $10 \%$ de la superficie con el adelanto de la edad al primer servicio de las vaquillonas permitió reducir la IE hasta un $17 \%$. Los resultados sugieren que es posible adoptar tecnologías disponibles para alcanzar los objetivos de reducción de IE. Aunque se requiere información experimental complementaria, este estudio provee información de utilidad referida a GEI para los tomadores de decisiones, políticos y productores, de modo de favorecer mayor promoción/adopción de tecnologías al tener en cuenta su respuesta económica.

Palabras clave: bovino carne, tecnología, huella de carbono, mitigación.

\section{ABSTRACT}

The aim of this study was to evaluate the productive economic performance and greenhouse gas emissions (GHG) in typical cow-calf farms in the Flooding Pampas, Argentina. A dynamic, bio-economic and environmental "whole-farm" model (SIMUGAN) and regional data were used to evaluate two contrasting scenarios obtained from the 4 systems identified in a previous study. Two scenarios were taken as baseline: medium-scale traditional farmer $(\mathrm{Tr})$ and large-scale local technified enterprise (Te). Both scenarios were intensified with the inclusion of two levels of fescue 
pasture under good management practices ( 5 or $10 \%$ of the farm area), and the anticipation of the first service of heifers (from 27 to 15 months age) for the scenarios with $10 \%$ of fescue pasture. The GHG intensity emissions (IE) varied accordingly to the intensification level in a similar trend for $\mathrm{Tr}$ and Te scenarios (between 16.4 and $19.9 \mathrm{~kg} \mathrm{CO}_{2} \mathrm{eq} \mathrm{kg} \mathrm{LW}^{-1}$ ). The additive effect for the $10 \%$ of fescue pasture incorporation combined with early mating of heifers reduced IE up to $17 \%$. Results suggest that it is feasible to reduce IE by the adoption of available technologies. Although further experimental research is required, this study provides useful information about GHG for policy makers and farmers in order to promote a higher promotion/adoption of technologies based on their economic response.

Key words: beef cattle, technology, carbon footprint, mitigation.

\section{INTRODUCCIÓN}

La necesidad de cubrir la creciente demanda global de alimentos lleva asociada la preocupación por el cambio climático y la minimización del impacto ambiental, como la reducción de los gases de efecto invernadero (GEI) en los sistemas de producción (Godfray et al., 2010). La agricultura, ganadería, silvicultura y otros usos de la tierra aportan el 39\% a las emisiones nacionales (368 Mtn $\mathrm{CO}_{2}$ eq), donde el 20\% de la emisiones de este sector se atribuyen a la ganadería en el Inventario Nacional de GEI 2014 (MAyDS, 2017). En nuestro país, los sistemas de cría aportan el $85 \%$ de las GEI asociadas a la producción de carne bovina, donde el $52 \%$ de las emisiones se generan en zonas templadas de la Pampa Húmeda (FAO y NZAGRC, 2017). Los GEI provenientes de la ganadería son el metano $\left(\mathrm{CH}_{4}\right)$, el óxido nitroso $\left(\mathrm{N}_{2} \mathrm{O}\right)$ y el dióxido de carbono $\left(\mathrm{CO}_{2}\right)$ que tienen $\mathrm{su}$ origen en diferentes fuentes del sistema productivo (Faverin et al., 2014).

La modelación de los sistemas de producción (whole-farm modelling) ha demostrado ser una valiosa herramienta para explorar oportunidades de innovación y cuantificación de impactos potenciales a esa escala (Berger et al., 2017). Recientemente FAO y NZAGRC (2017) evaluaron estrategias de mitigación del $\mathrm{CH}_{4}$ entérico y de heces $(62 \%$ de las emisiones nacionales de GEI provenientes de los sistemas de producción de carne bovina) donde, por ejemplo se analizaron mejoras en la cantidad y calidad de alimento, en la salud del rebaño (rodeo), respuesta reproductiva y dinámica del rodeo con oportunidades de mitigación costo eficientes. No obstante es relevante representar y analizar integralmente interacciones potenciales que incluyan $\mathrm{NO}_{2}$ y $\mathrm{CO}_{2}$ en estudios de modelación (Del Prado et al., 2013).

La Pampa Deprimida es la zona de cría más importante del país, con la mitad del stock de la Provincia de Buenos Aires (48\%) y produce anualmente más de 2,5 millones de terneros (MinAgro, 2015). Estos sistemas son de bajos insumos y se basan principalmente en pastizales naturales complementados con verdeos (forrajes anuales) y pasturas en diferente proporción (Arelovich et al., 2011; Faverin y Machado, 2018). La producción de carne en estos sistemas fluctúa entre 70-80 kg ha- ${ }^{-1}$ año ${ }^{-1}$, aunque los productores más eficientes pueden alcanzar producciones de $150 \mathrm{~kg} \mathrm{ha}^{-1}$ año $^{-1}$ (Arzubi, 2015; Faverin y Machado, 2018), lo cual denota que existen posibilidades de mejora de la eficiencia de estos sistemas con una transferencia de tecnología efectiva. La incorporación de pasturas asociadas a buenas prácticas de manejo (BPM), que abarca desde la siembra, mantenimiento y la utilización, es una de las propuestas tecnológicas destacadas para la región (Agnusdei y Di Marco, 2015). Las especies forrajeras ampliamente utilizadas son agropiro (Thinopyrum ponticum) y festuca (Festuca arundinacea) (Cid et al., 2008; Cid et al., 2011). La festuca puede lograr tasas de producción razonables de forraje de alta calidad (Lattanzi et al., 2007; Berger et al., 2014). Una mejora en la oferta forrajera podría no sólo tener efectos positivos per se, sino también adelantar la edad al primer servicio de las vaquillonas, promoviendo mejoras productivas y reducción de la intensidad de emisión (IE) de GEI por unidad de producto (Nguyen et al., 2013) al reducir la presencia de categorías improductivas en el rodeo (Beauchemin et al., 2010).

En un estudio de clasificación comparativa de sistemas de cría bovina de la Pampa Deprimida (Faverin y Machado, 2018) se identificaron 4 tipos de establecimientos. Como una primera aproximación, se observó la existencia de un sistema tradicional (Grupo 2) y tres tipos de sistemas tecnificados (Grupos 1, 3 y 4). En general, las tipologías no solo pueden reflejar la heterogeneidad de los establecimientos de una forma más precisa, sino también ser el punto de partida para representar los sistemas ganaderos base y valorar integralmente distintas estrategias de innovación, como el caso de pasturas BMP.

En este estudio se plantearon dos objetivos: a) simular dinámicamente los indicadores 
productivos, económicos y de IE de GEI/huella de carbono a nivel de dos tipos de establecimientos contrastantes de la Pampa Deprimida, y b) evaluar integralmente los cambios en los mismos indicadores cuando se incorporan algunas propuestas tecnológicas recomendadas regionalmente.

\section{MATERIALES Y MÉTODOS}

El estudio se ubica en la Pampa Deprimida ${ }^{1}$, región de clima templado y húmedo, con veranos e inviernos bien definidos, y heladas en invierno y primavera. El período libre de heladas es de 200260 días (Otondo, 2011). La temperatura promedio anual varía entre $14-16^{\circ} \mathrm{C}$ y las precipitaciones disminuyen al sur-oeste (1000-700 $\mathrm{mm}$ anuales). Las sequías son frecuentes en verano, período caracterizado por altas temperaturas y vientos fuertes. Basados en estudios previos de tipologías por Faverin y Machado (2018), en relación a recursos forrajeros, variables productivas y sociales, para este trabajo se seleccionaron los 2 escenarios más contrastantes productivamente según escala, base forrajera (Tabla 1) y porcentaje de destete $(69 \%$ vs. $81 \%)$. Estos escenarios (Tabla 1) se denominaron Productores tradicionales de escala media (en adelante Tr) y Empresa local tecnificada de escala grande (en adelante Te). A partir de Tr y Te (utilizados como sistemas base de las simulaciones), se evaluaron tres niveles adicionales de intensificación basados en la mejora de la oferta forrajera con 5 y $10 \%$ de la superficie total de pasturas de festuca con BPM ( $\operatorname{Tr} 5 \%, \operatorname{Te} 5 \%$, $\operatorname{Tr} 10 \%$ y $\mathrm{Te} 10 \%$, respectivamente). Adicionalmente se incluyeron dos planteos con $10 \%$ de pasturas BMP más el adelanto del servicio de las vaquillonas a los 15 meses de edad ( $\operatorname{Tr} 10 \% 15 \mathrm{~m}$ y Te10\%15m) (Tabla 1).

Se utilizó un modelo de simulación biofísico a nivel de establecimiento ganadero denominado SIMUGAN (Machado et al., 2010). SIMUGAN es un modelo web de dinámica diaria, basado en criterios/reglas de manejo y en computación distribuida para una mayor poder de cálculo (Arroqui et al., 2015). El modelo, implementado en Java, OpenLazlo (www.openlaszlo.org) y Spring Framework (www.springframework. org), opera espacialmente a nivel de parcelas de pastoreo dentro de potreros y animales dentro de rodeos(rebaños), y está estructurado en tres módulos o subsistemas principales, uno biofísico, uno de reglas de manejo y uno económico. Para cada escenario ganadero se simuló diariamente un año calendario (01 julio-30 junio) acorde a lo realizado en estudios previos (Berger et al., 2017). Las tasas de acumulación forrajera aérea ( $\mathrm{kg}$ MS $h^{-1} \mathrm{~d}^{-1}$ ) de los recursos forrajeros zonales, se obtuvieron del monitoreo satelital de pasturas y verdeos provistos por AACREA e INTA (LARTFAUBA, 2016). Los índices espectrales como el índice de vegetación normalizado (IVN) son obtenidos a partir de sensores remotos y considerados, en general, buenos estimadores de la productividad primaria neta aérea (Grigera et al., 2007). Las tasas de crecimiento mensuales de festuca BPM fueron extraídas de Berger et al. (2017) tomando el valor para un año promedio. La siembra de avena (Avena sativa) se realizó a fin de verano y se consumió de fines de marzo a septiembre. El sorgo (Sorghum bicolor) diferido doble propósito azucarado y/o silero azucarado se utilizó para la alimentación de las vacas en invierno, en el periodo abril a julio (Recavarren, 2007). Las producciones medias anuales, calidad, vida útil, régimen y tasas de fertilización, y el uso de combustible fósil por recurso se muestran en la Tabla 2.

El pastoreo fue rotativo diario y la asignación forrajera se basó en valores mensuales asumidos (entre 3 y $11 \mathrm{~kg} \mathrm{MS/100} \mathrm{kg} \mathrm{PV} \mathrm{d}{ }^{-1}$ ). La masa forrajera (MF) de los establecimientos (MF promedio, $\mathrm{kg}$ MS ha- ${ }^{-1}$ en un momento dado para todos los potreros) fue monitoreada semanalmente y se vinculó a reglas de manejo como las aplicadas por Berger et al. (2017). En principio, el modelo compara la MF con la masa mínima definida para cada mes (en promedio: $1660 \mathrm{~kg} \mathrm{MS} \mathrm{ha-1}$ para otoño-invierno y $1830 \mathrm{~kg}$ primavera-verano). SIMUGAN dispone de una regla de masa forrajera que identifica los desvíos entre la MF actual del sistema $(\mathrm{kg} \mathrm{MS} \mathrm{ha-1),} \mathrm{con}$ una MF objetivo promedio para cada mes que le otorga flexibilidad al manejo, incrementando o disminuyendo la asignación forrajera inicialmente definida, y así permite recuperar o disminuir MF (Machado et al., 2010). A partir de los datos simulados se estimó la intensidad de pastoreo (IP) $(\mathrm{kg}$ forraje consumido/kg forraje producido) que representa el nivel de utilización forrajera a partir de la relación entre proporción de la masa forrajera consumida con la disponible para pastorear en momento determinado, tomando valores mínimos y máximos entre 0 y 1 (Petz et al., 2014).

El margen bruto (MB) (U\$S ha ${ }^{-1}$ año $^{-1}$ ) definido por la diferencia entre ingresos netos (venta de terneros/as y vacas de descarte) y los

\footnotetext{
${ }^{1}$ Cuenca del Salado (CS) 6,5 millones de ha, y Depresión de Laprida (DL) 2,12 millones de ha, en el área de influencia de las Estaciones Experimentales (EEA) del INTA, Cuenca del Salado y una zona de la EEA INTA Balcarce llamado Territorio Centro Oeste, acorde a lo descripto por Faverin y Machado (2018)
} 
costos directos (AACREA, 1990) (implantación y mantenimiento de pasturas, implantación de verdeos, mano de obra, sanidad del rodeo) se estimó (de Julio a Junio) utilizando la información de precios de la revista Márgenes Agropecuarios (Arbolave, 2017). Adicionalmente se calculó la relación de ingreso neto a costos directos (Ingreso/Costo) según FAO y NZAGRC (2017). Cada planteo consideró un empleado permanente y los empleados temporarios de $7 \mathrm{y}$
12 jornales año ${ }^{-1}$ para los planteos tradicionales y para tecnificados, respectivamente. El nivel de la prevención sanitaria aplicada fue acorde a Arbolave (2016).

Para la estimación de las emisiones de GEI $\left(\mathrm{CH}_{4}, \mathrm{~N}_{2} \mathrm{O}, \mathrm{CO}_{2}\right)$ se incorporaron al modelo SIMUGAN ecuaciones que representan las distintas fuentes de emisión dentro de sistema productivo "hasta la tranquera" o "cradle to farm-gate", con un enfoque de análisis de ciclo de

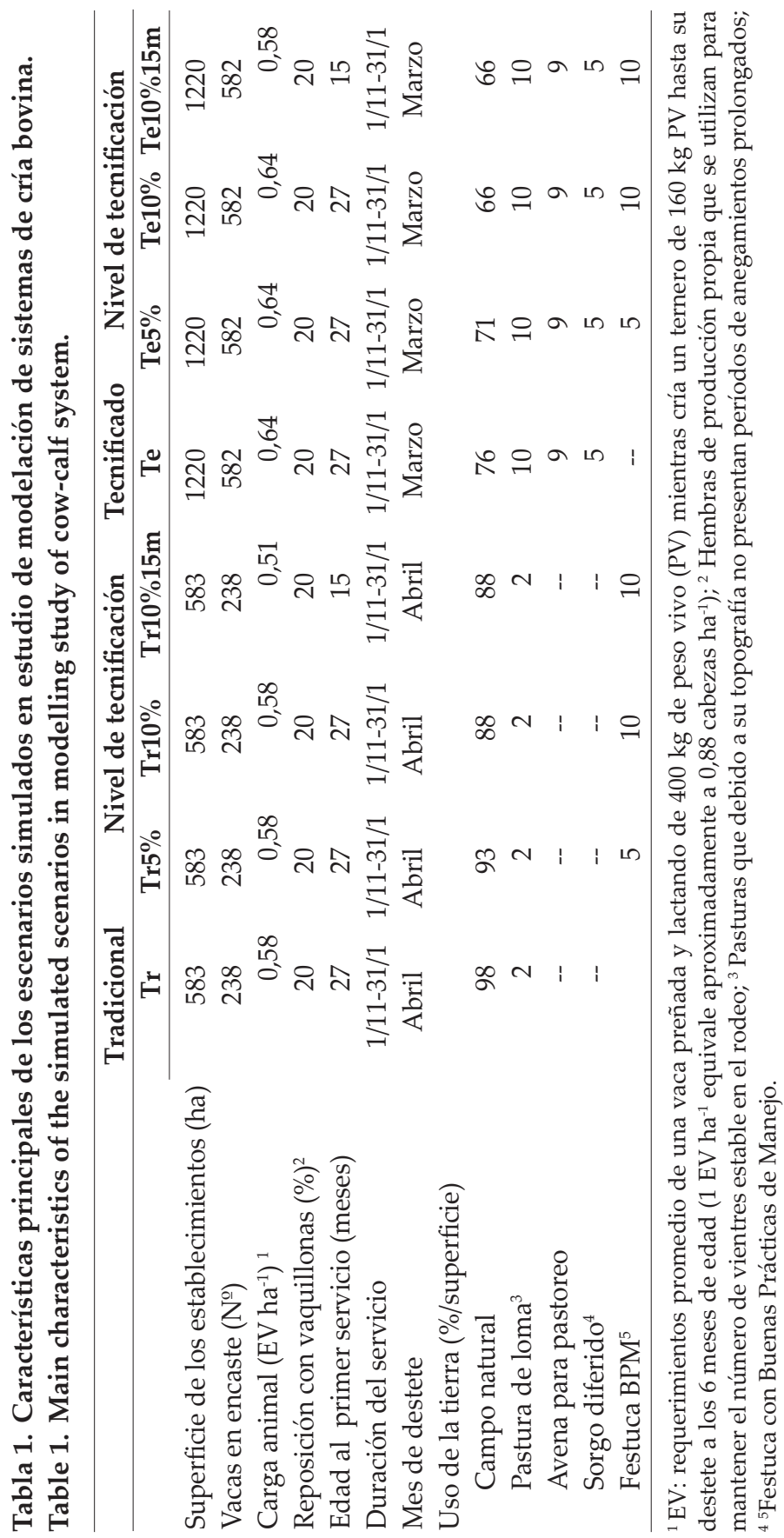


vida (Guinée et al., 2002). Este enfoque relaciona el impacto ambiental de un determinado sistema de producción a una unidad funcional (UF), la cual representa el principal producto del sistema analizado, definida en este estudio como $\mathrm{kg}$ de peso vivo (PV) producido. Las emisiones de
GEI se expresaron en unidades de dióxido de carbono equivalente $\left(\mathrm{CO}_{2} \mathrm{eq}\right)$ teniendo en cuenta el potencial de calentamiento global (PCG) de cada gas (IPCC, 2006) asumiendo un horizonte temporal de 100 años. Los PCG fueron 25, 298 y 1 para el $\mathrm{CH}_{4}, \mathrm{~N}_{2} \mathrm{O}$ y $\mathrm{CO}_{2}$, respectivamente

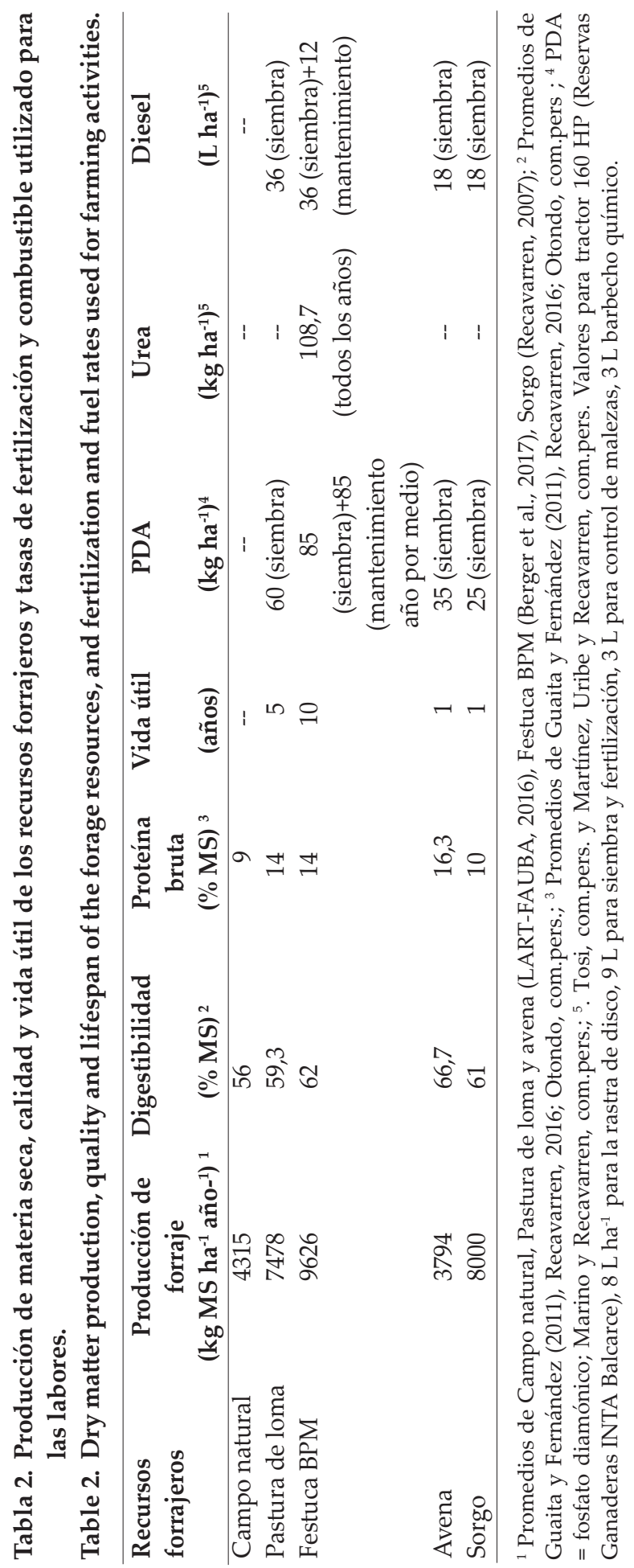


(Forster et al., 2007). A partir de las estimaciones diarias de consumo diario de MS estimadas por el SIMUGAN por potrero y categoría animal, se estimó la energía bruta consumida que fue utilizada para estimar las emisiones de $\mathrm{CH}_{4}$ atribuibles a la fermentación entérica y a las heces depositadas en pastoreo mediante ecuaciones del IPCC (IPCC, 2006-Nivel 2). Las emisiones de $\mathrm{N}_{2} \mathrm{O}$ directas e indirectas se estimaron a partir de la orina y heces depositadas durante el pastoreo, de residuos agrícolas y de fertilizantes nitrogenados sintéticos (IPCC, 2006- Nivel 1). En el primer caso, se estimaron el nitrógeno excretado a partir del consumo diario de MS y el contenido de nitrógeno de la dieta, insumos necesarios para las estimaciones de esta fuente. Las emisiones de $\mathrm{N}_{2} \mathrm{O}$ a partir de la aplicación de fertilizantes sintéticos se estimaron a partir de la fertilización nitrogenada aplicada anualmente a los recursos forrajeros, y la cantidad anual de $\mathrm{N}$ en los residuos agrícolas devueltos a los suelos (IPCC, 2006- Nivel 1). En éste último caso, fue necesario contar con el rendimiento de cultivos que es tomado como insumo en el SIMUGAN y, en el caso de las pasturas se consideró como insumo la producción total de forraje.

Las emisiones de $\mathrm{CO}_{2}$ se estimaron a partir del uso de combustibles fósiles (República Argentina, 2005) y de la ruptura de la urea en los establecimientos (IPCC, 2006). Las emisiones consideradas fuera de los límites de los establecimientos provinieron de la producción, embalaje, almacenamiento y transporte de fertilizantes sintéticos basados en N y P (Becoña et al., 2014), de la producción, embalaje, almacenamiento y transporte de la energía para producir herbicidas (Becoña et al., 2014), de la producción de combustible diesel (Wang, 2007; Chianese et al., 2009) y de la producción de semillas (Rotz et al., 2012). Se estimaron las IE (kg $\mathrm{CO}_{2}$ eq $\mathrm{kg} \mathrm{PV}^{-1}$ año ${ }^{-1}$ ) y las emisiones de GEI por unidad de superficie (E/ha: $\mathrm{kg} \mathrm{CO}_{2}$ eq ha- $\mathrm{año}^{-1}$ ).

\section{RESULTADOS}

Las menores producciones de carne se registraron en el planteo base $\operatorname{Tr}$ y sus alternativas de intensificación (Tabla 3). Las diferencias relativas en incrementos en producción de carne por efecto de la intensificación con respecto a los planteos base $\operatorname{Tr}$ y Te fueron 17 al 23\%. También por efecto de la intensificación, el porcentaje de terneros destetados varió entre 67 y $81 \%$ para $\mathrm{Tr}$, y entre 78 a $81 \%$ para $\mathrm{Te}$, y en ambos casos alcanzó su mayor valor en los planteos con $10 \%$ de festuca BPM y servicio de vaquillonas a los 15 meses (Tabla 3). El MB se incrementó en un 17,4 y 1,7\% al aumentar el nivel de intensificación de
$\operatorname{Tr}$ y Te, respectivamente, atribuible en el último caso a un aumento mayor relativo en los costos directos en los ingresos (Tabla 3).

Como resultado de la intensificación de $\mathrm{Tr}$, la $\mathrm{E} / \mathrm{h}$ a fue menor que en los planteos que resultan de intensificar a Te (Tabla 3). No obstante, cuando se comparó dentro de ambos tipos de establecimientos, las menores $\mathrm{E} / \mathrm{ha}$ se lograron cuando se incorporó $10 \%$ de festuca BPM y se realizó el encaste de las vaquillonas a los 15 meses ( $\operatorname{Tr} 10 \% 15 \mathrm{~m}$ o Te $10 \% 15 \mathrm{~m}$, Tabla 3). Las diferencias se debieron fundamentalmente a las menores emisiones de $\mathrm{CH}_{4}$, provenientes principalmente de la fermentación entérica (1091 $\mathrm{kg} \mathrm{CO}_{2}$ eq ha ${ }^{-1}$ para $\operatorname{Tr}$ vs. $1033 \mathrm{~kg} \mathrm{CO}_{2}$ eq ha- ${ }^{-1}$ para $\operatorname{Tr} 10 \% 15 \mathrm{~m}$ y $1298 \mathrm{~kg} \mathrm{CO}_{2}$ eq ha-1 para Te vs. $1203 \mathrm{~kg} \mathrm{CO}_{2}$ eq ha- ${ }^{-1}$ para Te $\left.10 \% 15 \mathrm{~m}\right)$, y de $\mathrm{N}_{2} \mathrm{O}$ de las heces y orina depositadas durante el pastoreo $(426 \mathrm{~kg}$ $\mathrm{CO}_{2}$ eq ha ${ }^{-1}$ para $\mathrm{Tr}$ vs. $441 \mathrm{~kg} \mathrm{CO}_{2}$ eq ha- ${ }^{-1}$ para $\operatorname{Tr} 10 \% 15 \mathrm{~m}$ y $524 \mathrm{~kg} \mathrm{CO}_{2}$ eq ha ${ }^{-1}$ para Te vs. $517 \mathrm{~kg}$ $\mathrm{CO}_{2}$ eq ha-1 para $\left.\mathrm{Te} 10 \% 15 \mathrm{~m}\right)$. El $\mathrm{CH}_{4}$ disminuyó su participación en las emisiones a medida que se intensificó de 71,9 a $66,3 \%$ y de 68,3 a $63,2 \%$ en $\mathrm{Tr}$ y $\mathrm{Te}$, respectivamente; en tanto, el $\mathrm{N}_{2} \mathrm{O}$ incrementó su participación (27,9 a 31,5\% y 30,4 a $33,2 \%$ para $\operatorname{Tr}$ y $\mathrm{Te}$, respectivamente). $\mathrm{El} \mathrm{CO}_{2}$ representó menos del 3,6\% en todos los planteos, fundamentalmente asociado a las labores y el uso y compra de insumos necesarios para la implementación de la superficie de festuca BPM atribuibles al laboreo y a la compra de insumos necesarios para esta actividad.

Las emisiones de GEI por unidad de producto variaron según el nivel de intensificación de forma similar para Tr y Te (entre 16,4 y 19,9 $\mathrm{kg} \mathrm{CO}_{2}$ eq kg $\mathrm{PV}^{-1}$ ) (Tabla 3). Las IE fueron mayores en $\operatorname{Tr} \mathrm{y} \mathrm{Te}, \mathrm{y}$ disminuyeron del 16,6 y 14,6\% respectivamente, cuando se incrementó la superficie destinada a festuca BPM (5,5 y 5,2\%, respectivamente) y principalmente cuando se adelantó la edad al primer servicio de las vaquillonas (11,1 y $9,4 \%$, respectivamente). El mayor aporte a las IE provino del $\mathrm{CH}_{4}$ (proveniente principalmente de la fermentación entérica) que disminuyó su contribución entre 71,9 a $66,2 \%$ desde $\operatorname{Tr}$ a $\operatorname{Tr} 10 \% 15 \mathrm{~m}$ y entre 68,2 a $63,2 \%$ desde Te a Te10\%15m (Tabla 3). Por otro lado, las emisiones de $\mathrm{N}_{2} \mathrm{O}$ aumentaron entre 27,9 a $31,5 \%$ para los planteos para los planteos originados en $\operatorname{Tr} y$ entre 30,3 a $33,2 \%$ en el caso de $\mathrm{Te}$, atribuibles principalmente a las provenientes de la orina y heces depositadas por animales en pastoreo. El $\mathrm{CO}_{2}$ emitido por unidad de producto fue menor al 3,7\% para todos los planteos simulados.

Los planteos $\operatorname{Tr} 10 \%$ y $\operatorname{Tr} 10 \% 15 \mathrm{~m}$ más productivos, con mayor porcentaje de destete y menor IP, presentaron una $\mathrm{E} / \mathrm{ha}$ similar a $\mathrm{Tr}$, pero con menor IE, mayor $\mathrm{MB}$ y un menor ingreso/ 
costo (Fig. 1). En el caso de los planteos Te10\% y Te $10 \% 15 \mathrm{~m}$ más productivos, tuvieron un mejor porcentaje de destete y menor IP que el planteo base, E/ha similares al planteo Te, menores IE pero sin una mejora del $\mathrm{MB}$ asociado a mayores costos neutralizan los mayores ingresos (Tabla 2 y Fig. 2).

\section{DISCUSIÓN}

Estos resultados corresponden al primer estudio sobre estimación de huella de carbono/
IE a nivel establecimiento (hasta la tranquera) en sistemas típicos de cría de la Pampa Deprimida. El enfoque dinámico utilizado con el modelo SIMUGAN (Machado et al., 2010) y la incorporación de las ecuaciones principalmente desarrolladas por el IPCC (IPCC, 2006) permitieron una valoración integral de los efectos potenciales de la intensificación de establecimientos típicos de cría bovina acorde a metodologías recomendadas internacionalmente (Crosson et al., 2011).

Las IE halladas en nuestro estudio (Tabla

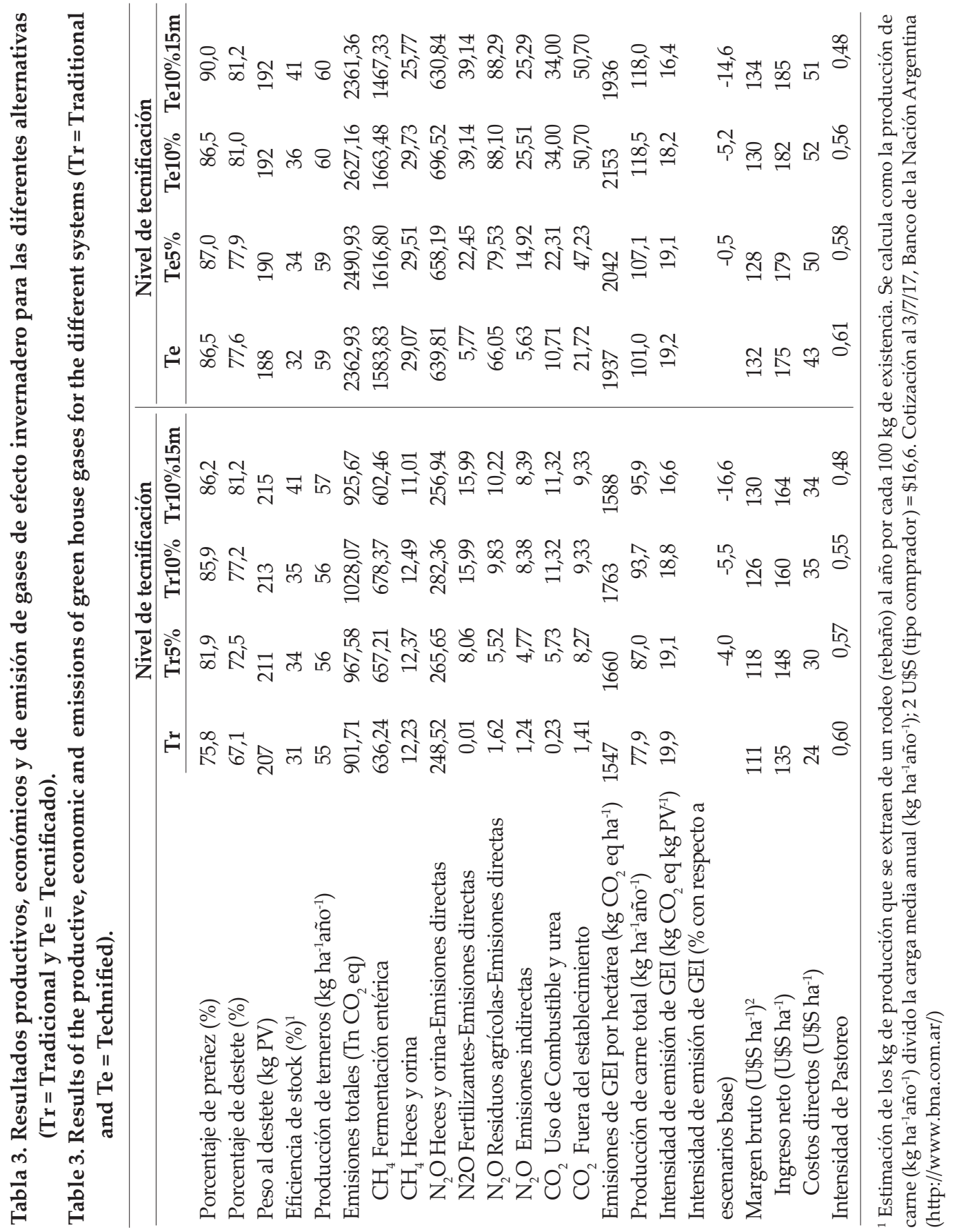




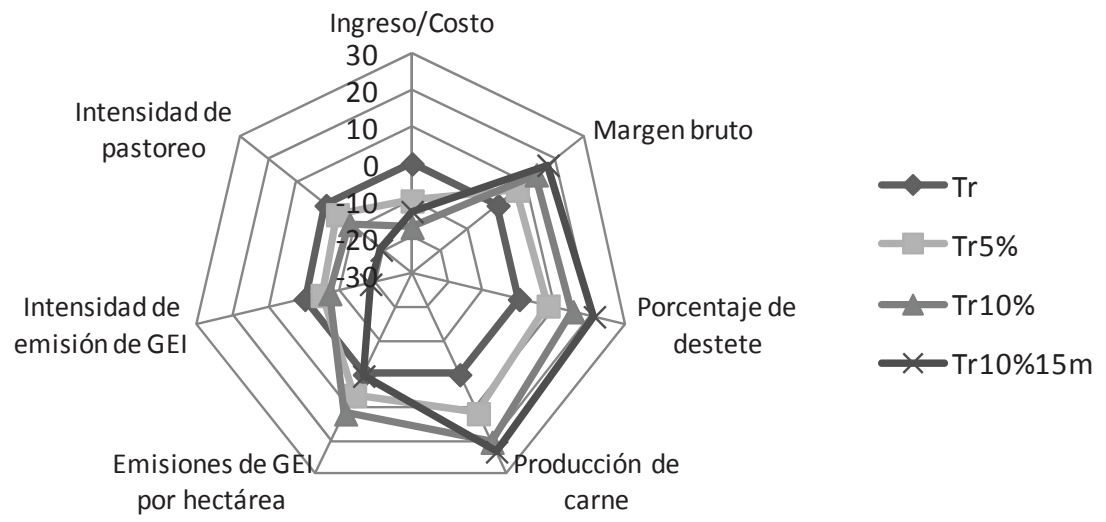

Fig. 1. Indicadores productivos, económicos y de emisiones para escenarios tradicionales. El gráfico de radar indica los porcentajes de cambio relativo respecto al escenario base (Tr), Tr: Planteo Base; Tr5\% y Tr10\%: 5 y 10\% de la superficie total con festuca BPM, respectivamente; Tr10\%15m: $10 \%$ de total superficie con festuca BPM y primer servicio de las vaquillonas a los 15 meses.

Fig, 1. Productive, economic and emission indicators for traditional scenarios. The radar chart indicates the percentages of relative change with respect to the base scenario $(\operatorname{Tr}), \operatorname{Tr}$ : Base scenario; $\operatorname{Tr} 5 \%$ and $\operatorname{Tr} 10 \%$ : 5 and $10 \%$ of total area with BPM fescue, respectively; $\operatorname{Tr} 10 \% 15 \mathrm{~m}$ : $10 \%$ of the total area with BPM fescue and first heifers bred at 15 months of age.

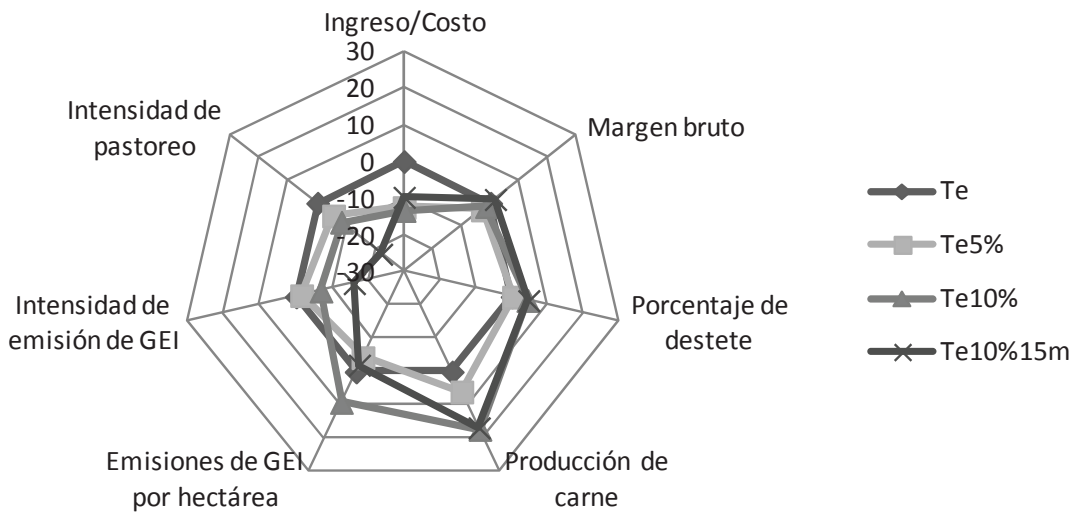

Fig. 2. Indicadores productivos, económicos y de emisiones para escenarios tecnificados. El gráfico de radar indica los porcentajes de cambio relativo respecto al escenario base (Te). Te: Planteo Base; $\mathrm{Te} 5 \%$ y $\mathrm{Te} 10 \%: 5 \%$ y $10 \%$ de la superficie total con festuca BPM, respectivamente; Te10\%15m: 10\% de total superficie con festuca BPM y primer servicio de las vaquillonas a los 15 meses.

Fig. 2. Productive, economic and emission indicators for technical scenarios, The radar chart indicates the percentages of relative change with respect to the base scenario (Te), Te: Base scenario; Te5\% and Te10\%: 5 and 10\% of total area with BPM fescue, respectively; Te10\% 15m: 10\% of the total area with BPM fescue and first heifers bred at 15 months of age.

3) fueron menores a los $30 \mathrm{~kg} \mathrm{CO}_{2}$ eq $\mathrm{kg} \mathrm{PV}^{-1}$ informados por FAO y NZAGRC (2017) para establecimientos de cría de la región pampeana de Argentina, pero en el rango de estudios realizados en otros países. Becoña et al. (2014) señalan una gran variabilidad entre establecimientos de Uruguay donde la alimentación se basó en campo natural, pasturas mejoradas o implantadas y verdeos anuales de invierno. Los promedios de E/ ha y IE fueron de $2030 \mathrm{~kg} \mathrm{CO}_{2}$ eq ha $^{-1}$ (rango: 1490 $2827 \mathrm{~kg} \mathrm{CO}_{2}$ eq ha $^{-1}$ ) y 20, $8 \mathrm{~kg} \mathrm{CO}_{2}$ eq kg PV (rango: $^{-1}$ 11,4-32,2 $\mathrm{kg} \mathrm{CO}_{2}$ eq kg PV-1), respectivamente. En un trabajo previo, Becoña et al. (2013) informaron los primeros valores para el país vecino $(1895 \mathrm{~kg}$ $\mathrm{CO}_{2}$ eq ha ${ }^{-1}$ y $18,4 \mathrm{~kg} \mathrm{CO}_{2}$ eq $\mathrm{kg} \mathrm{PV}^{-1}$ ) para un caso de un sistemas de cría con $90 \%$ de destete. Picasso 
et al. (2014), también para Uruguay, mencionan IE promedio de $21,9 \mathrm{~kg} \mathrm{CO}_{2}$ eq $\mathrm{kg} \mathrm{PV}^{-1}$ para sistemas pastoriles con productividades similares a las del presente estudio, donde nuestros planteos base resultaron 9 a 12\% menores. La dispersión entre los datos bibliográficos y la diversidad de sistemas productivos (por ej. para la región Pampeana definieron 9 sistemas de producción para el inventario nacional de GEI) (MAyDS, 2017), evidencia la importancia de estudios complementarios.

La estrategia para incrementar la producción $\mathrm{y}$ calidad del forraje mediante festuca BPM (Agnudei y Di Marco, 2015) permitió además reducir las IE (hasta un $6 \%$ en $\operatorname{Tr}$ y Te con respecto a los escenarios base), aumentar el número de terneros destetados y la eficiencia de producción en los sistemas de cría aún cuando sólo un 5\% de la superficie se destinó a la implantación de festuca BPM (Tabla 3; Figs. 1 y 2). La mejora en la calidad del forraje es considerada una estrategia de mitigación (Beauchemin et al., 2011; Gerber et al., 2013), al permitir reducir el tiempo del alimento en el rumen, potenciar la digestión postruminal y disminuir la proporción de la energía que es convertida en $\mathrm{CH}_{4}$ (Waghorn and Hegarty, 2011).

La segunda fuente más importante de emisión en los sistemas de cría evaluados fue la orina y las heces depositadas durante el pastoreo (Tabla 3) que resulta en un perfil típico de los sistemas de producción de la región (Becoña et al., 2013; Picasso et al., 2014; MAyDS, 2017). Chadwick et al. (2011) mencionan que las heces y orina pueden ser una fuente substancial de $\mathrm{N}_{2} \mathrm{O}$, mientras que la cantidad de $\mathrm{CH}_{4}$ es exigua como en nuestro estudio (Tabla 3). Un mayor consumo de MS por animal y secundariamente, un leve aumento proteico de la dieta asociado a la festuca BPM (Tabla 2), podrían explicar el incremento en el ingreso de $\mathrm{N}$ urinario y de heces al suelo que incrementaron las $\mathrm{E} / \mathrm{ha}$ en los planteos tradicionales y tecnificados. Sin embargo, las IE incorporando todos los GEI fueron menores a medida que se incluyeron mejoras en los sistemas de producción (Tabla 3 ).

Similar a lo observado por Andreini y Place (2014), adelantar a 15 meses el servicio de las vaquillonas permitió reducir porcentualmente las IE en 11,1 y $9,4 \%$ para $\operatorname{Tr} 10 \% 15 \mathrm{~m}$ y $\mathrm{Te} 10 \% 15 \mathrm{~m}$, respectivamente (Tabla 3) con porcentajes de cambio de 16,6 y $14,6 \%$ con respecto a $\operatorname{Tr}$ y Te, respectivamente (Figs. 1 y 2). En este sentido, Andreini y Place (2014) mencionaron que adelantar el desarrollo de las vaquillonas y reducir la edad al primer parto pueden aumentar la eficiencia de la producción y disminuir la IE debido a una reducción en el consumo de alimento y emisiones de GEI. En este caso, la reducción de las IE en nuestros resultados se logró combinando la potencialidad de estrategias de carácter aditivo (Beauchemin et al., 2011), como lo son la mejora de la base forrajera y la reducción del tiempo al primer servicio.

La incorporación de pequeñas superficies de festuca BPM permitió no solo aumentar la producción y adelantar el servicio de las vaquillonas, sino también obtener un mayor $\mathrm{MB}$ en los planteos de mejora para $\mathrm{Tr}$, pero no en el caso de Te (Tabla 3). Por lo tanto, la implementación de estrategias de mitigación en este último caso puede resultar per se poco atractivas económicamente para los productores (Browne et al., 2011), lo cual debiera contemplarse en el diseño de posibles políticas diferenciales de mitigación de GEI.

El incremento en la producción forrajera con los mismos niveles de carga animal significó una reducción de la IP de 0,6 a 0,4 entre planteos bases y tecnificados, con incrementos en la producción de carne por una mayor respuesta individual y una reducción en la IE. Petz et al. (2014) sugieren mantener niveles de IP entre 0,4 y 0,6 para favorecer diferentes servicios ecosistémicos de las pasturas, como el secuestro de carbono, evitar la erosión y la pérdida de biodiversidad en sistemas de base pastoril. Aunque este estudio no contempla esas variables, deja las bases para el diseño de estudios complementarios como por ejemplo, la reducción en la superficie de verdeos a expensas de un incremento en las pasturas perennes manejadas con buenas practicas, que podría contribuir adicionalmente a la mitigación de GEI por un incremento del carbono edáfico (Rotz, 2018).

\section{CONCLUSIONES}

Los resultados de simulación demostraron que las estrategias de mitigación evaluadas pueden disminuir potencialmente las emisiones por unidad de producto en establecimientos típicos de cría bovina de la Pampa Deprimida. El efecto aditivo de dichas estrategia, implicó mejoras graduales en los resultados productivos y económicos para sistemas tradicionales, mientras que la menor respuesta diferencial obtenida en sistemas tecnificados requeriría la exploración de otras alternativas tecnológicas. Aunque se requiere información experimental complementaria, este estudio provee información de utilidad para decisores políticos y productores, de modo de favorecer mayor promoción/ adopción de tecnologías de mitigación de GEI al tener en cuenta su respuesta económica. 


\section{RECONOCIMIENTOS}

Este trabajo es parte de la tesis doctoral del primer autor. Los autores desean agradecer al INTA por el soporte financiero (Beca de Postgrado, BASUR-1272101, y PNNAT-1128023) y a la UNMDP (AGR 413/13). También queremos agradecer a los Ings. M.A. Marino, M.J. Uribe, J. Martínez y J.C. Tosi por sus aportes de información para las simulaciones, al Dr. Mauricio Arroquy por la implementación del submodelo de emisiones de GEI y al Ing. P. Recavarren por la información, comentarios y sugerencias.

\section{LITERATURA CITADA}

AACREA. 1990. Normas para medir los resultados económicos en las empresas agropecuarias. Convenio Asociación Argentina de Consorcios Regionales de Experimentación Agrícola (AACREA), Banco RIO, Buenos Aires, Argentina.

Agnusdei, M.G., y O. Di Marco. 2015. Más producción de carne, menos riesgo y más flexibilidad con pasturas perennes en suelos bajos. En Primer Premio Colombo \& Magliano 75을 Aniversario. Lara Producciones Eds., INTA, Buenos Aires, Argentina.

Andreini, E.M., and S.E. Place. 2014. Current approaches of beef cattle systems. Life cycle assessment: A review. White Paper: Sustainability. Cattlemen's Beef Board and National Cattlemen's Beef Association. Department of Animal Science, Oklahoma State University, Stillwater, USA.

Arbolave, M.R. 2016. Márgenes Agropecuarios. Suplemento Ganadero 2016, Buenos Aires, Argentina.

Arbolave, M.R. 2017. Márgenes Agropecuarios. Año 33 № 385, Julio 2017. Buenos Aires, Argentina.

Arelovich, H.M., R.D. Bravo, and M.F. Martínez. 2011. Development, characteristics, and trends for beef cattle production in Argentina. Animal Frontiers 1:37-45. doi:10.2527/ af.2011-0021. doi:10.2527/af.2011-0021.

Arroqui, M., J. Rodríguez, H. Vázquez, C. Machado, C. Mateos, and A. Zunino. 2015. JASAG: a gridification tool for agricultural simulation applications. Concurrency and Computation: Practice and Experience 27(17):4716-4740. doi:10.1002/cpe.3453.

Beauchemin, K.A., H.H. Janzen, S.M. Little, T.A. McAllister, and S.M. McGinn. 2010. Life cycle assessment of greenhouse gas emissions from beef production in western Canada: A case study. Agr. Syst. 103:371-379. doi:10.1016/j.agsy.2010.03.008.
Beauchemin, K.A., H.H. Janzen, T.A. McAllister, and S.M. McGinn. 2011. Mitigation of greenhouse gas emissions from beef production in western Canada - Evaluation using farm-based life cycle assessment. Anim. Feed Sci. Technol.166-167:663-677. doi:10.1016/j.anifeedsci.2011.04.047.

Becoña, G., S. Ledgard, and E. Wedderburn. 2013. A comparison of greenhouse gas emissions from Uruguayan and New Zealand beef systems. Agrociencia Uruguay 17:120-130.

Becoña, G., L. Astigarraga, and V.D. Picasso. 2014. Greenhouse gas emissions of beef cow-calf grazing systems in Uruguay. Sustain. Agri. Res. 3(2):89-105. doi:10.5539/sar.v3n2p89.

Berger, H., F. Bilotto, L. Bell, L., and C.F. Machado. 2017. Feedbase intervention in a cow-calf system in the flooding pampas of Argentina: 2 . Estimation of the marginal value of additional feed. Agr. Syst. 158:68-77. doi:10.1016/j. agsy.2017.09.004.

Berger, H., C.F. Machado, M. Agnusdei, and B.R. Cullen. 2014. Use of a biophysical simulation model (DairyMod) to represent tall fescue pasture growth in Argentina. Grass and Forage Science 69:441-453. doi: 10.1111/ gfs.12064.

Browne, N.A., R.J. Eckard, R. Behrendt, and R.S. Kingwell. 2011. A comparative analysis of on-farm greenhouse gas emissions from agricultural enterprises in south eastern Australia. Anim. Feed Sci. Technol.166:641-652. doi:10.1071/AN13543.

Cid, M.S., C.M. Ferri, M.A. Brizuela, and O. Sala. 2008. Structural heterogeneity and productivity of a tall fescue pasture grazed rotationally by cattle at four stocking densities. Grassland Science 54:9-16. doi: 10.1111/j.1744697X.2007.00099.x.

Cid, M.S., R.C. Fernández Grecco, M. Oesterheld, J.M. Paruelo, A.F. Cibils, and M.A. Brizuela. 2011. Grass-fed beef production systems of Argentina's flooding pampas. Understanding ecosystem heterogeneity to improve livestock production. Outlook Agr. 40(2):181-189. doi: 10.5367/oa.2011.0040.

Crosson, P., L. Shalloo, D. O’Brien, G.J. Lanigan, P.A. Foley, and T.M. Boland. 2011. A review of whole farm systems models of greenhouse gas emissions from beef and dairy cattle production systems. Anim. Feed Sci. Technol. 166:29-45. doi:10.1016/j.anifeedsci.2011.04.001.

Chadwick, D., S. Sommer, R. Thorman, D. Fangueiro, L. Cardens, B. Amon, et al. 2011. Manure management: Implications for greenhouse gas emissions. Anim. Feed Sci. Technol. 166-167:514-531. doi:10.1016/j. anifeedsci.2011.04.036. 
Chianese, D.S., C.A. Rotz, and T.L. Richard. 2009. Simulation of carbon dioxide emissions from dairy farms to assess greenhouse gas reduction strategies. Trans. ASABE 52:13011312.

Del Prado, A., P. Crosson, J.E. Olesen, and C.A. Rotz. 2013. Whole-farm models to quantify greenhouse gas emissions and their potential use for linking climate change mitigation and adaptation in temperature grassland ruminant-based farming systems. Animal 7(s2):373-385. doi:10.1017/ S1751731113000748.

Faverin, C., R. Gratton, y C.F. Machado. 2014. Emisiones de gases de efecto invernadero en sistemas de producción de carne vacuna de base pastoril. Revisión bibliográfica. Rev. Arg. Prod. Anim. 34(1):33-54.

Faverin, C., y C.F. Machado. 2018. Tipologías y caracterización de sistemas de cría bovina de la Pampa Deprimida. Chilean J. Agric. Sci. 35(1):3-13

FAO, and NZAGRC. 2017. Low-emissions development of the beef cattle sector in Argentina. Reducing enteric methane for food security and livelihoods. Food and Agriculture Organization of the United Nations (FAO). New Zealand Agricultural Greenhouse Gas Research Centre (NZAGRC), Rome, Italy.

Forster, P., V. Ramaswamy, P. Artaxo, T. Berntsen, R. Betts, D.W. Fahey, et al. 2007. Changes in atmospheric constituents and in radiative forcing. Chapter 2. p. 131-234. In Solomon, S.; Qin, D.; Manning, M.; Chen, Z.; Marquis, M.; Averyt, K.B., et al. (eds.) Climate Change 2007: The Physical Science Basis. Contribution of Working Group I to the Fourth Assessment Report of the Inter Governmental Panel on Climate Change. Cambridge University Press, Cambridge, United Kingdom/New York, NewYork, USA.

Gerber, P.J., H. Steinfeld, B. Henderson, A. Mottet, C. Opio, J. Dijkman, et al. 2013. Hacer frente al cambio climático a través de la ganadería. Evaluación global de las emisiones y las oportunidades de mitigación. 129 p. Organización de las Naciones Unidas para la Alimentación y la Agricultura. FAO, Roma, Italia.

Godfray, C.J., J.R. Beddington, I.R. Crute, L. Haddad, D. Lawrence, J.F. Muir, et al. 2010. Food security: The challenge of feeding 9 billion people. Science 327:812-818.

Grigera, G., M. Oesterheld, and F. Pacin. 2007. Monitoring forage production for farmers' decision making. Agr. Syst. 94:637-648. doi:10.1016/j.agsy.2007.01.001
Guaita, M.S., y H.H. Fernández. 2011. Tablas de composición química de los alimentos para rumiantes. 60 p. Ediciones INTA, Balcarce, Argentina.

Guinée, J.B., M. Gorrée, R. Heijungs, G. Huppes, R. Kleijn, A. De Koning, et al. 2002. Life cycle assessment. An operational guide to the ISO standards. Centre of Environmental Science, Leiden University, Leiden, The Netherlands.

IPCC. 2006. IPCC guidelines for national greenhouse gas inventories. Intergovernmental. Panel on Climate Change, IGES, Cambridge University Press, Cambridge, England.

Lattanzi, F.A., A. Mazzanti, and M.H. Wade. 2007. Seasonal animal production of temperate and mediterranean tall fescue cultivars under continuous variable stocking with close control of sward state. Aust. J. Agr. Res. 58:203-213. doi: 10.1071/AR06164.

LART-FAUBA. 2016. Sistema de Seguimiento Forrajero. Buenos Aires, Argentina. Disponible en http://lart.agro.uba.ar/ (Consulta 15 febrero 2018).

Machado, C.F., S.T. Morris, J. Hodgson, M.A. Arroqui, and P.A. Mangudo. 2010. A webbased model for simulating whole-farm beef cattle systems. Comput. Electron. Agr. 74:129136. doi:10.1016/j.compag.2010.07.007.

MAyDS. 2017. Inventario de Gases de Efecto Invernadero de Argentina. Ministerio de Ambiente y Desarrollo Sustentable (MAyDS), Presidencia de la Nación. 36 p. Disponible en https://inventariogei.ambiente.gob.ar/ files/inventario-nacional-gei-argentina.pdf (Consultado 15 febrero 2018)

MinAgro. 2015. Caracterización de la Producción Bovina, Buenos Aires - Corrientes - Chaco - Formosa - La Pampa - Misiones. 193 p. Sistema de Monitoreo del Sector de la Carne Bovina. Serie 1. Ministerio de Agroindustria, Presidencia de la Nación, Subsecretaría de Ganadería. INTA, SENASA, Argentina

Nguyen, T.T.H., M. Doreau, M. Eugene, M.S.Corson, F. Garcia-Launay, G. Chesneau, et al. 2013. Effect of farming practices for greenhouse gas mitigation and subsequent alternative land use on environmental impacts of beef cattle production systems. Animal 7-5:860-869. doi: 10.1017/ S1751731112002200.

Otondo, J. 2011. Efectos de la introducción de especies megatérmicas sobre características agronómicas y edáficas de un ambiente halomórfico de la Pampa Inundable. Tesis Magister Scientiae. Universidad de Buenos Aires, Área Recursos Naturales, FAUBA, Argentina. 
Petz, K., R. Alkemade, M. Bakkenes, C.J.E. Schulp, M. Van Der Velde, and R. Leemans. 2014. Mapping and modelling trade-offs and synergies between grazing intensity and ecosystem services in rangelands using global-scale datasets and models. Global Environ. Change 29:223-234. doi:10.1016/j. gloenvcha.2014.08.007.

Picasso, V.D., P.D. Modernel, G. Becoña, L. Salvo, L. Gutiérrez, and L. Astigarraga. 2014. Sustainability of meat production beyond carbon footprint: a synthesis of case studies from studies from grazing systems in Uruguay. Meat Sci. 98:346-354. doi: /10.1016/j.meatsci.2014.07.005.

Recavarren, P. 2007. Sorgos diferidos como reserva forrajera invernal en rodeos de cría de la zona de la Depresión de Laprida, Balcarce, Argentina. Disponible en http:// www.agrositio.com/vertext/vertext. php?id $=82451 \&$ se $=36$. (Consulta 28 junio 2018)

Recavarren, P. 2016. La producción agropecuaria en Olavarría, Benito Juárez, Laprida y Gral. La Madrid: evolución y desafíos a futuro. Balcarce, Buenos Aires. Ediciones INTA. Disponible en https://inta.gob.ar/ sites/default/files/inta_la_produccion_ agropecuaria_en_olavarria_juarez_laprida_ la_madrid_2016.pdf (Consulta 15 febrero 2018)
República Argentina. 2005. Inventario de gases de efecto invernadero de la República Argentina - Año 2000. Tomo II. 377 p. Fundación Bariloche, Buenos Aires, Argentina.

Rotz, C.A., M.S. Corson, D.S. Chianese, F. Montes, S.D. Hafner, and C.U. Coiner. 2012. Integrated farm system model: Reference manual. USDA Agricultural Research Service, University Park, Pennsylvania. Available at www. ars. usda.gov/SP2UserFiles/Place/19020000/ ifsmreference.pdf (Accessed 15 Feb. 2018).

Rotz, C.A. 2018. Modeling greenhouse gas emissions from dairy farms. J. Dairy Sci. 101:1-16. doi: 10.3168/jds.2017-13272.

Waghorn, G.C., and R.S. Hegarty. 2011. Lowering ruminant methane emissions through improved feed conversion efficiency. Animal Feed Science and Technology 166-167:291301. doi: 10.1016/j.anifeedsci.2011.04.019.

Wang, M. 2007. GREET. Version 1.8a. Argonne National Laboratory, Argonne, IL. Available

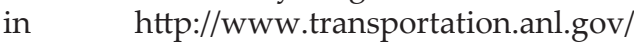
software/GREET/ (Consulta 15 febrero 2018) 